\title{
Global Regulation on microRNA in Hepatitis B Virus-Associated Hepatocellular Carcinoma
}

\author{
Angela M. Liu,, ${ }^{1,2}$ Chunsheng Zhang, ${ }^{3,4}$ Julja Burchard, ${ }^{3,5}$ S.T. Fan, ${ }^{2}$ Kwong-Fai Wong,,2 \\ Hongyue Dai, ${ }^{3,4}$ Ronnie T. Poon, ${ }^{2}$ and John M. Luk ${ }^{1,2}$
}

\begin{abstract}
Recent work has revealed the causative links between deregulation of microRNAs (miRNAs) and cancer development. In hepatocellular carcinoma (HCC), aberrant expression of miRNAs has been observed, but the molecular mechanisms that contribute to such changes remains to be elucidated. Here, we reported the analysis of miRNA expression in 94 pairs of tumor and adjacent nontumor tissues from HBV-associated HCC in Chinese patients. We found miRNAs were aberrantly expressed in HCC tissues. To investigate the cause of such deregulation, we detected changes in DNA copy number by measuring locus-specific hybridization intensity, and found changes in expression of several miRNAs are correlated with genomic amplification or deletion. For example, the genomic regions of miR-30d and miR-151 were amplified in $\sim 50 \%$ of HCC tumor tissues, and the expressions of these miRNAs are significantly correlated with DNA copy number. We also employed cDNA microarray data, and provide evidence that key regulators of the miRNA biosynthetic pathway, including DROSHA, DGCR8, AGO1, and AGO2, are frequently overexpressed in HCC. This study provides molecular clues that may contribute to the global changes of miRNA expression in HCC.
\end{abstract}

\section{Introduction}

$\mathbf{H}$ EPATOCELlular CARCINOMA (HCC) is a lethal malignancy with no effective interventions, and causes approximate 600,000 deaths each year (Parkin et al., 2005). The difficult clinical situation is due to lack of early diagnosis, limited options for treatments, and the heterogeneous nature of the disease. Different mechanisms contribute to the heterogeneity, including chromosomal abnormality, epigenetic regulation, and copy number variation. Recently, in addition to these mechanisms, microRNA (miRNA) deregulation has emerged as a new mechanism affecting HCC biology.

miRNAs are about 22-nucleotide long, noncoding RNAs that play an important role in regulating gene expression at posttranscriptional level (Lagos-Quintana et al., 2002). Growing evidence showed that they are involved in diverse cellular processes, including apoptosis, cell proliferation, stress resistance, and cell differentiation (Esquela-Kerscher and Slack, 2006). Given the wide impact of miRNAs, it is not surprising that a number of them are implicated in cancer. miRNAs are expressed abnormally in various types of cancer, including
HCC (Budhu et al., 2008; Foekens et al., 2008; Yanaihara et al., 2006). The underlying mechanism by which miRNA expression is deregulated in HCC has been unclear so far, although in some cancers it could be resulted in part from genomic amplification or deletion, mutation, and epigenetic silencing (Garzon et al., 2009). In this context, we employ the sensitive and accurate realtime quantitative-PCR (qPCR) detection method to profile 220 miRNAs in a cohort of 94 pairs of tumor and adjacent nontumor HCC tissues. We explore the mechanisms that contribute to the aberrant expression of miRNAs and performed integrated analysis of single nucleotide polymorphism (SNP) array data and mRNA expression data of enzymes involved in miRNA biogenesis. We found that in some instances, miRNA expression is correlated with DNA copy number, and enzymes involved in miRNA biogenesis are frequently deregulated in HCC.

\section{Materials and Methods}

\section{Clinical samples}

Human HCC samples were collected from patients who had undergone hepatectomy for curative treatment of HCC at

\footnotetext{
${ }^{1}$ Department of Pharmacology, Department of Surgery, and Cancer Science Institute, National University of Singapore, Singapore.

${ }^{2}$ Department of Surgery, The University of Hong Kong, Queen Mary Hospital, Hong Kong Special Administrative Region, People's Republic of China.

${ }^{3}$ Rosetta Inpharmatics LLC, a wholly owned subsidiary of Merck and Co., Inc., Seattle, Washington.

${ }^{4}$ Merck Research Laboratories, Boston, Massachusetts.

${ }^{5}$ Sirna Therapeutics, Inc., San Francisco, California.
} 
Queen Mary Hospital, Pokfulam, Hong Kong, between 1990 and 2007. Resected tumor and adjacent nontumor liver tissues were obtained as described (Hao et al., 2009). Informed consents were obtained from patients regarding the use of the liver specimens for research. Demographic and clinicopathologic features are as described (Burchard et al., 2010).

\section{RNA extraction}

The milled human liver tissue samples were homogenized in cryopreservation tubes with a vortex mixer after addition of TRIzol (Invitrogen, Carlsbad, CA, USA). The total RNA was further purified using the Promega SV-96 total RNA kit (Promega Corp., Madison, WI, USA), and then assayed by Agilent Bioanalyzer (Agilent Technologies, Santa Clara, CA, USA) for quality. The yield was assayed by Ribogreen metrics (Invitrogen Corp.).

\section{$\operatorname{miRNA}$ and $c D N A$ profiling}

miRNA profiling was performed using custom made quantitative PCR assays as described (Raymond et al., 2005). cDNA profiling was performed using custom Affymetrix array, RM-HU01Aa520485 RSTA Custom Affymetrix 1.0. Hybridization, labeling and scanning of microarrays were performed according to the manufacturer's recommendations (Affymetrix Inc., Santa Clara, CA, USA) (Burchard et al., 2010).

\section{Copy number variation (CNV) analysis}

$\mathrm{CNV}$ was measured using the Illumina platform that contains a $650 \mathrm{~K}$ array, and the results are expressed as the $\log \mathrm{R}$ ratio (that is, the intensity ratio of the studied sample to a number of reference samples). The genomic locations of each differentially expressed miRNA and the single-nucleotide polymorphism probes that flanked in the miRNA genomic regions were identified. $\mathrm{CNV}$ of the miRNAs were obtained by averaging the $\log \mathrm{R}$ ratio from those corresponding singlenucleotide polymorphism probes. The same procedure was used to study the CNV of all the 220 miRNAs. In addition to analysis of the continuous CNV data, a hidden Markov model
(HMM) was applied to determine if there was copy number gain or loss as described previously (Liu et al., 2009).

\section{Results \\ Genomic amplification and deletion of miRNAs}

We analyzed the miRNA expression levels in 94 pairs of HCC tissues and corresponding adjacent nontumor tissues. A subset of miRNAs was differentially expressed. Given the changes in miRNA expression in HCC, it is important to understand how these changes occur. We first tested if the changes in miRNA expression are due to genomic amplification or deletion. We analyzed the SNP data from the same set of samples, and determined whether the expression of the miRNAs was associated with DNA copy number. We found that nine differentially expressed miRNAs were significantly correlated with DNA copy number with $p$-value $<0.05$ after Bonferroni correction and were amplified or deleted in genomes of tumor samples (Table 1). These miRNAs were transcribed from seven distinct chromosomal loci. The genomic loci contain two miRNA clusters, including miR-30e-3p and miR-30c located at 1q34.2, also miR-18a and miR-19a located at 13q31.3. We found that miR-30d and miR-151, both located at chromosome 8 , have high correlation with DNA copy number $(r=0.6$ and 0.7 , respectively; Pearson correlation, and $p<0.0001)$. These two miRNAs were significantly overexpressed in HCC patients, and the genomic regions of these two miRNAs were amplified in $\sim 50 \%$ of HCC patients $(p<0.0005)$. Thus, the comparison of $\mathrm{CNV}$ with expression data suggests that genomic alteration is one of the mechanisms that contribute to the miRNA deregulation.

\section{Changes in miRNA biosynthesis may contribute to deregulation of miRNA expression}

Recent studies suggested that proteins involved in miRNA biosynthesis are deregulated in cancer, such as lung cancer (Karube et al., 2005) and ovarian cancer (Merritt et al., 2008). Therefore, we tested whether genes required for miRNA biosynthesis were differentially expressed in our HCC tissue samples. The expression level of genes DROSHA, DGCR8,

TAble 1. CORRElation of MiRNA EXPRESSION AND DNA COPY NUMber (MiRNAs INDiCATED in Red Are Transcribed from Multiple Loci)

\begin{tabular}{|c|c|c|c|c|c|c|c|}
\hline miRNA & $\begin{array}{c}\text { Correlation } \\
\text { with DNA copy number }\end{array}$ & p-value & $\begin{array}{l}\text { Deletion } \\
\text { rate }\end{array}$ & p-value & $\begin{array}{l}\text { Amplification } \\
\text { rate }\end{array}$ & $\mathrm{p}$-value & $\begin{array}{l}\text { miRNA Alteration } \\
\text { (frequency, \%) }\end{array}$ \\
\hline \multicolumn{8}{|l|}{ Chromosome 1} \\
\hline miR-34A & 0.3823 & 0.0002 & 0.4205 & $<0.0001$ & - & - & Up (65.6) \\
\hline miR-30E-3P & 0.5336 & $<0.0001$ & 0.2500 & 0.0450 & - & - & Down (81.3) \\
\hline miR-30C & 0.4620 & $<0.0001$ & 0.2500 & 0.0450 & - & - & Down (74.0) \\
\hline \multicolumn{8}{|l|}{ Chromosome 8} \\
\hline miR-30D & 0.6111 & $<0.0001$ & - & - & 0.5568 & 0.0004 & Up (76.0) \\
\hline miR-151 & 0.7068 & $<0.0001$ & - & - & 0.5227 & 0.0004 & Up (92.7) \\
\hline \multicolumn{8}{|l|}{ Chromosome 13} \\
\hline miR-18A & 0.4009 & 0.0001 & 0.2614 & 0.0331 & - & - & Up (92.7) \\
\hline miR-19A & 0.5046 & $<0.0001$ & 0.2614 & 0.0331 & - & - & Up (67.7) \\
\hline $\begin{array}{l}\text { Chromosome } 14 \\
\text { miR-345 }\end{array}$ & 0.4488 & $<0.0001$ & 0.2841 & 0.0210 & - & - & Up (75.0) \\
\hline $\begin{array}{l}\text { Chromosome } 17 \\
\text { miR-21 }\end{array}$ & 0.3732 & 0.0003 & - & - & 0.2841 & 0.0138 & Up (90.6) \\
\hline
\end{tabular}


DICER1, AGO1, AGO2, AGO3, and AGO4 were analyzed. We found that DROSHA, DGCR8, AGO1, and AGO2, were significantly overexpressed, whereas DICER1 and AGO3 were significantly downregulated in HCC tumor tissues than in adjacent nontumor tissues (Fig. 1a). In addition, the expression levels of these genes were more variable in HCC tumor tissues. Among the genes required for biosynthesis of miRNAs, DROSHA was the most differentially expressed. It is responsible for producing pre-miRNAs and thus may affect the miRNA expression levels. We looked for miRNAs that are positively correlated with DROSHA expression, and found that seven miRNAs (miR-324-5p, -301, -222, -106a, -18a, -93, and $-106 b$ ) are positively correlated with DROSHA (Pearson correlation $>0.3$ and $p$-value $<0.05$ after Bonferroni correction) (Fig. 1b). The result suggests that changes in expression of genes involved in miRNA biosynthesis may have an impact on the expression levels of miRNAs in HCC.

\section{Discussion}

We found that the distinct miRNA signature of HCC tumor is at least in part attributed to the frequent CNV (either gain or loss) in cancer genomes. We showed that expression levels of several miRNAs are highly correlated with either amplifi- cation or deletion of the chromosomal regions from where they are encoded. For example, the genomic regions of miR$30 \mathrm{~d}$ and miR-151 were amplified significantly in 56 and 52\%, respectively, in our clinical cohorts. A recent study reported that the genomic region of miR-151 is frequently amplified in HCC (Ding et al., 2010), which is in accordance with our results in this report. In addition, miR-21, which is often found overexpressed in various types of cancer, is in a genomic region amplified in $28 \%$ of our clinical cohorts. It has been shown that in chronic lymphocytic leukemia (CLL), CNV influences expression of miRNAs. The genomic region encoding miR-15 and miR-16 is deleted in more than $50 \%$ of the patients with CLL (Calin et al., 2002), and these miRNAs have been shown to have tumor suppressing activities in a leukemic xenograft model (Calin et al., 2008). It should be note that the genomic region of miR-34a was deleted at a high rate of $42 \%$ in our patient cohort; however, it was overexpressed in $\sim 65 \%$ of the tumor samples. Recent studies have reported the inhibitory effect of miR-34a on tumor growth (Hermeking, 2007; Wiggins et al., 2010). Tumors with lower miR-34a expression due to genomic deletion might have more aggressive phenotype. This subset of tumors is worthy of further investigation to enhance our understanding of the CNV action on oncogenesis. $\mathrm{CNV}$ has been widely known to shape the a
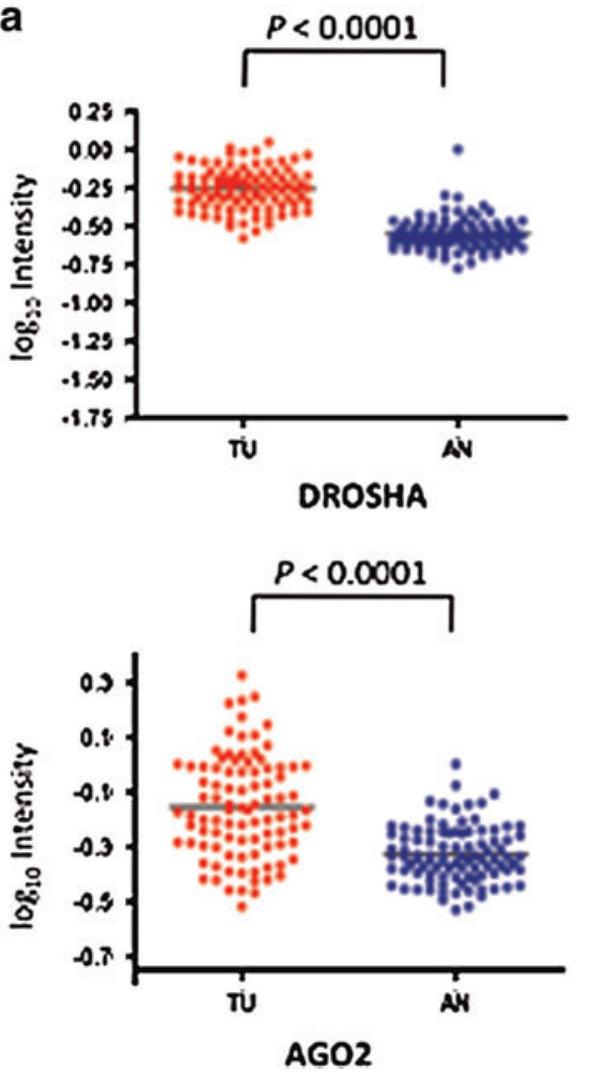
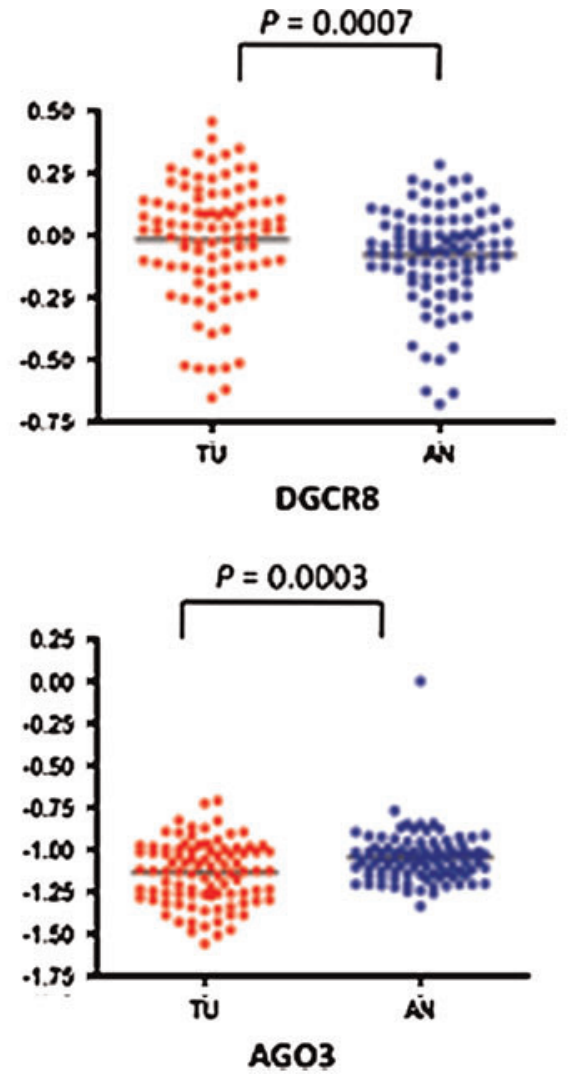
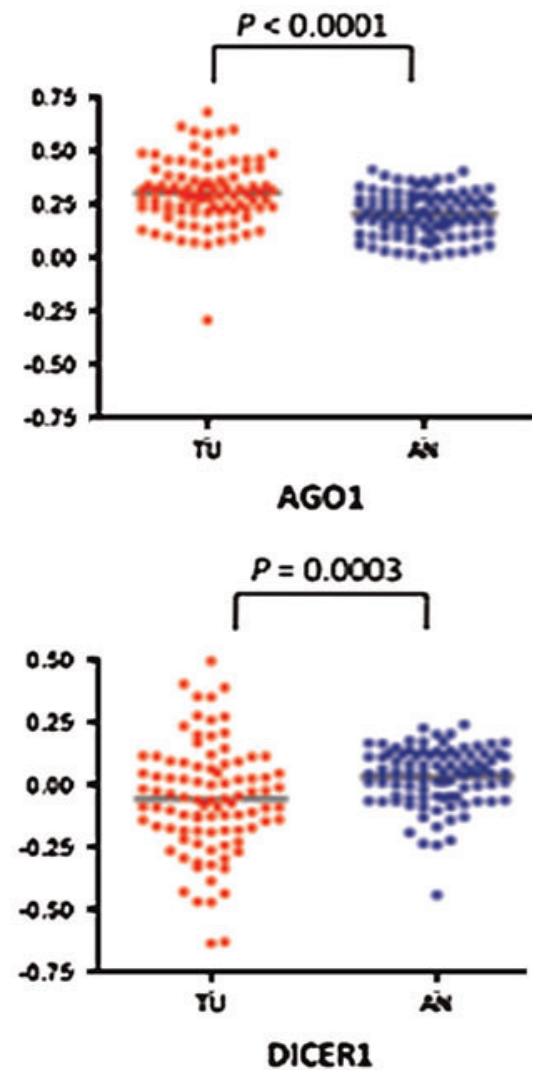

FIG. 1. Genes required for miRNA biosynthesis are differentially expressed between tumor and nontumor tissues. (a) Scatter plots of $\log _{10}$ intensity of DICER1, DGCR8, AGO1, AGO2, AGO3, and DROSHA between tumor (TU) and adjacent nontumor (AN) tissues. Differential expression was assessed by Student $t$-test. (b) Shown are the expression levels of the seven miRNAs positively correlated with DROSHA expression in tumor and adjacent nontumor tissues from 94 patients. Left panel: the blue dots indicate whether the tissue is tumor (Yes) or adjacent nontumor (No). Center panel: a heat map showing the $\log 10$ ratio of expression level of each individual tissue sample to the mean of all tissue samples. Right panel: normalized DROSHA intensity. 
b

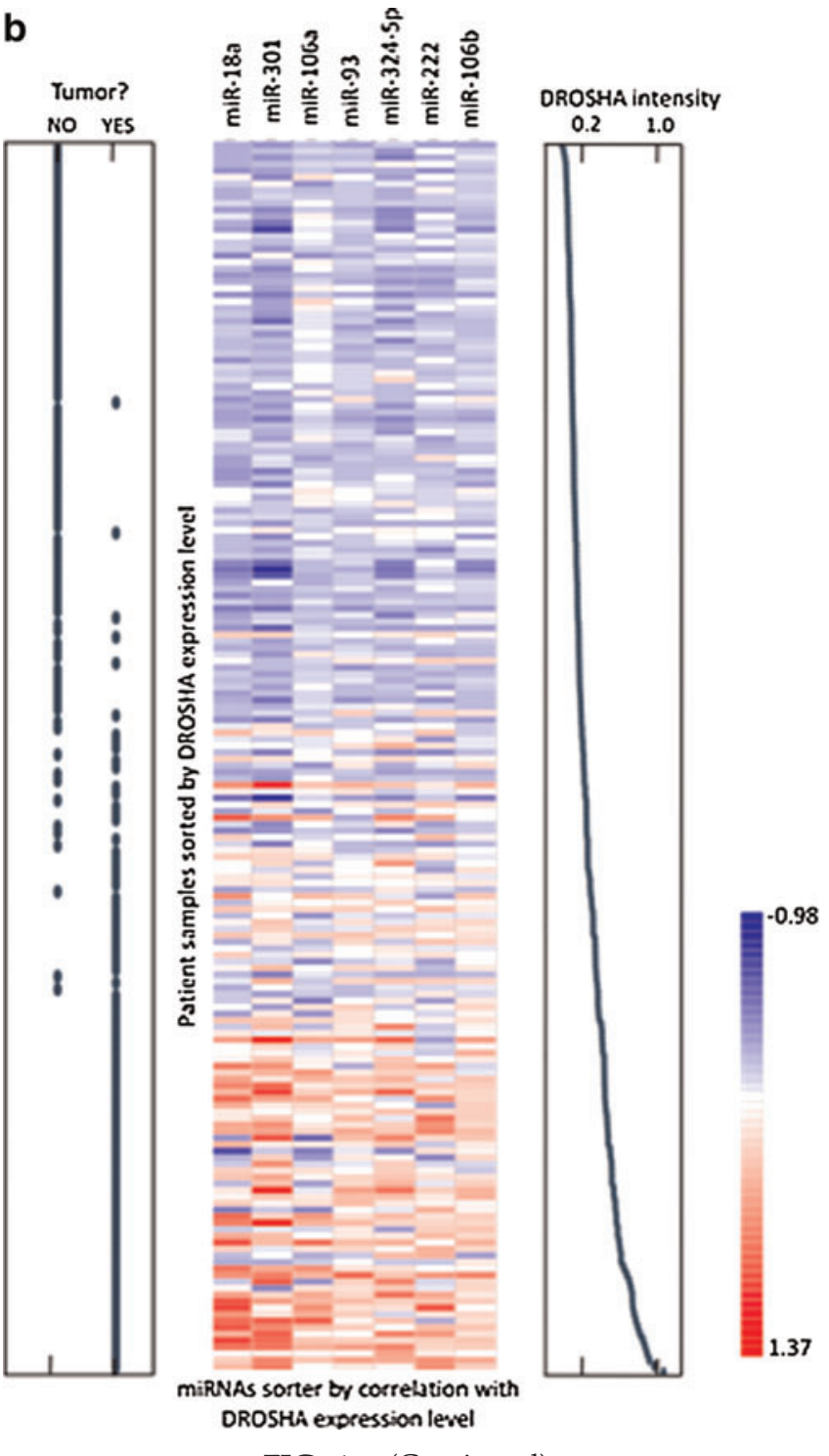

FIG. 1. (Continued).

transcriptome with its direct effect on gene expression (Cooper et al., 2008). We herein suggested that CNV also influences HCC transcriptome through indirect or secondary action via miRNAs.

In addition to the $\mathrm{CNV}$ causal effects, miRNA expression in HCC is also affected by deregulated biogenesis. This kind of deregulation has been observed in several types of solid cancers including lung (Muralidhar et al., 2007), ovarian (Flavin et al., 2008), cervical carcinomas (Muralidhar et al., 2007), and breast cancer (Grelier et al., 2009), but remains unknown in HCC. In our study, we found DROSHA was significantly overexpressed in HCC tumor tissues. The overexpression of DROSHA has also been reported in ovarian (Flavin et al., 2008) and cervical carcinomas (Muralidhar et al., 2007). We found seven miRNAs highly correlated with DROSHA expression. Several of these miRNAs have been reported to have oncogenic properties. For example, miR-106b and miR-93 were overexpressed in HCC tumor tissues and have a role in cell proliferation and anchorageindependent growth (Li et al., 2009). On the other hand, the downregulation of DICER1 expression was observed in our clinical cohort. In lung cancer, the lower expression of DICER1 promoted tumorigenesis in vitro and in mouse model (Kumar et al., 2007), whereas in ovarian cancer, low DICER1 expression was linked to poor overall survival (Faggad et al., 2010). Together, the data show that key components of the miRNA biosynthetic pathway emerge to play important roles in cancer development.

Changes in miRNA expression were complex. By investigating the SNP and mRNA data, we suggested that the CNV, and changes in the expression of miRNA biosynthetic pathway components may have pivotal role of contributing to the aberrant expression of miRNAs in HCC. This study may provide hints to further research on the molecular basis for understanding changes in miRNA expression in the development of HCC.

\section{Author Disclosure Statement}

The authors declare no competing financial interest in this study.

\section{References}

Budhu, A., Jia, H.L., Forgues, M., Liu, C.G., Goldstein, D., Lam, A., et al. (2008). Identification of metastasis-related microRNAs in hepatocellular carcinoma. Hepatology 47, 897-907.

Burchard, J., Zhang, C., Liu, A.M., Lee, N.P., Wong, K.F., Sham, P.C., et al. (2010). miR-122 as a regulator of mitochondrial metabolic gene network in hepatocellular carcinoma. Mol Syst Biol 6, 402.

Calin, G.A., Dumitru, C.D., Shimizu, M., Bichi, R., Zupo, S., Noch, E., et al. (2002). Frequent deletions and down-regulation of micro-RNA genes miR15 and miR16 at 13q14 in chronic lymphocytic leukemia. Proc Natl Acad Sci USA 99, 1552415529.

Calin, G.A., Cimmino, A., Fabbri, M., Ferracin, M., Wojcik, S.E., Shimizu, M., et al. (2008). MiR-15a and miR-16-1 cluster functions in human leukemia. Proc Natl Acad Sci USA 105, 5166-5171.

Cooper, G.M., Johnson, J.A., Langaee, T.Y., Feng, H., Stanaway, I.B., Schwarz, U.I., et al. (2008). A genome-wide scan for common genetic variants with a large influence on warfarin maintenance dose. Blood 112, 1022-1027.

Ding, J., Huang, S., Wu, S., Zhao, Y., Liang, L., Yan, M., et al. (2010). Gain of miR-151 on chromosome 8q24.3 facilitates tumour cell migration and spreading through downregulating RhoGDIA. Nat Cell Biol 12, 390-399.

Esquela-Kerscher, A., and Slack, F.J. (2006). Oncomirs-microRNAs with a role in cancer. Nat Rev Cancer 6, 259-269.

Faggad, A., Budczies, J., Tchernitsa, O., Darb-Esfahani, S., Sehouli, J., Muller, B.M., et al. (2010). Prognostic significance of Dicer expression in ovarian cancer-link to global microRNA changes and oestrogen receptor expression. J Pathol 220, 382-391.

Flavin, R.J., Smyth, P.C., Finn, S.P., Laios, A., O'Toole, S.A., Barrett, C., et al. (2008). Altered eIF6 and Dicer expression is associated with clinicopathological features in ovarian serous carcinoma patients. Mod Pathol 21, 676-684.

Foekens, J.A., Sieuwerts, A.M., Smid, M., Look, M.P., De Weerd, V., Boersma, A.W., et al. (2008). Four miRNAs associated with aggressiveness of lymph node-negative, estrogen receptorpositive human breast cancer. Proc Natl Acad Sci USA 105, 13021-13026.

Garzon, R., Calin, G.A., Croce, C.M. (2009). MicroRNAs in cancer. Annu Rev Med 60, 167-179. 
Grelier, G., Voirin, N., Ay, A.S., Cox, D.G., Chabaud, S., Treilleux, I., et al. (2009). Prognostic value of Dicer expression in human breast cancers and association with the mesenchymal phenotype. Br J Cancer 101, 673-683.

Hao, K., Luk, J.M., Lee, N.P., Mao, M., Zhang, C., Ferguson, M.D., et al. (2009). Predicting prognosis in hepatocellular carcinoma after curative surgery with common clinicopathologic parameters. BMC Cancer 9, 389.

Hermeking, H. (2007). p53 enters the microRNA world. Cancer Cell 12, 414-418.

Karube, Y., Tanaka, H., Osada, H., Tomida, S., Tatematsu, Y., Yanagisawa, K., et al. (2005). Reduced expression of Dicer associated with poor prognosis in lung cancer patients. Cancer Sci 96, 111-115.

Kumar, M.S., Kumar, M.S., Lu, J., Lu, J., Mercer, K.L., Mercer, K.L., et al. (2007). Impaired microRNA processing enhances cellular transformation and tumorigenesis. Nat Genet 39, 673.

Lagos-Quintana, M., Rauhut, R., Yalcin, A., Meyer, J., Lendeckel, W., Tuschl, T. (2002). Identification of tissue-specific microRNAs from mouse. Curr Biol 12, 735-739.

Li, Y., Tan, W., Neo, T.W., Aung, M.O., Wasser, S., Lim, S.G., et al. (2009). Role of the miR-106b-25 microRNA cluster in hepatocellular carcinoma. Cancer Sci 100, 1234-1242.

Liu, L.X., Lee, N.P., Chan, V.W., Xue, W., Zender, L., Zhang, C., et al. (2009). Targeting cadherin-17 inactivates Wnt signaling and inhibits tumor growth in liver carcinoma. Hepatology 50, 1453-1463.

Merritt, W.M., Lin, Y.G., Han, L.Y., Kamat, A.A., Spannuth, W.A., Schmandt, R., et al. (2008). Dicer, Drosha, and outcomes in patients with ovarian cancer. N Engl J Med 359, 2641-2650.

Muralidhar, B., Goldstein, L.D., Ng, G., Winder, D.M., Palmer, R.D., Gooding, E.L., et al. (2007). Global microRNA profiles in cervical squamous cell carcinoma depend on Drosha expression levels. J Pathol 212, 368-377.

Parkin, D.M., Bray, F., Ferlay, J., Pisani, P. (2005). Global cancer statistics, 2002. CA: Cancer J Clin 55, 74-108.

Raymond, C.K., Roberts, B.S., Garrett-Engele, P., Lim, L.P., Johnson, J.M. (2005). Simple, quantitative primer-extension PCR assay for direct monitoring of microRNAs and shortinterfering RNAs. RNA 11, 1737-1744.

Wiggins, J.F., Ruffino, L., Kelnar, K., Omotola, M., Patrawala, L., Brown, D., et al. (2010). Development of a lung cancer therapeutic based on the tumor suppressor microRNA-34. Cancer Res 70, 5923-5930.

Yanaihara, N., Caplen, N., Bowman, E., Seike, M., Kumamoto, K., Yi, M., et al. (2006). Unique microRNA molecular profiles in lung cancer diagnosis and prognosis. Cancer Cell 9, 189-198.

Addres correspondence to: Dr. John Luk

Department of Pharmacology NUHS, National University of Singapore

10 Medical Drive, MD11 117597 Singapore

E-mail: jmluk@nus.edu.sg

OR

Dr. Ronnie Poon Department of Surgery University of Hong Kong Pokfulam, Hong Kong

E-mail: poontp@hku.hk 
